\title{
PREDICTION OF VORTEX-INDUCED VIBRATION OF FLEXIBLE RISER USING AN IMPROVED WAKE-OSCILLATOR MODEL
}

\author{
Chen Wein-min* \\ Division of Engineering Sciences \\ Research, Institute of Mechanics, \\ Chinese Academy of Sciences, \\ Beijing 100190, China
}

\author{
Zhang Liwu \\ Division of Engineering Sciences \\ Research, Institute of Mechanics, \\ Chinese Academy of Sciences, \\ Beijing 100190, China
}

\author{
Li Min \\ School of Aeronautics Sciences \\ and Technology, Beijing \\ University of Aeronautics and \\ Astronautics, Beijing 100191, \\ China
}

\begin{abstract}
Based on improving the wake-oscillator model, an analytical model for vortex-induced vibration (VIV) of flexible riser under non-uniform current is presented, in which the variation of added mass at lock-in and the nonlinear relationship between amplitude of response and reduced velocity are considered. By means of empirical formula combining iteration computation, the improved analytical model can be conveniently programmed into computer code with simpler and faster computation process than CFD so as to be suitable to application $f$ practical engineering. This model is validated by comparing with experimental result and numerical simulation. Our results show that the improved model can predict VIV response and lock-in region more accurately. At last, illustrative examples are given in which the amplitude of response of flexible riser experiencing VIV under action of non-uniform current is calculated and effects of riser tension and flow distribution along span of riser are explored. It is demonstrated that with the variation of tension and flow distribution, lock-in region of mode behaves in different way, and thus the final response is a synthesis of response of locked modes.
\end{abstract}

\section{INTRODUCTION}

More and more deepwater platforms such as TLP, SPAR and FPSO are used in offshore gas and oil exploration and production. When the depth of sea and the length of structure increase, the flexibility of the structure also increases. On the other hand, because ocean current and wave action are getting greater, interaction between fluid and structure becomes stronger. In particular, vortex-induced vibration (VIV) of risers and tension legs, especially in the lock-in (synchronization) range, has become a very challenging problem.
Many computation models such as wake-oscillator model, correlation model, statistic model and polynomial Galerkin model have been developed to calculate response of a circular cylinder undergoing VIV. Among these, the wake-oscillator model (Hartlen ${ }^{[1]}$ and Iwan ${ }^{[2]}$ ) benefiting from its reasonable physical mechanism, convenient computation process and satisfactory accuracy has been popularly applied to the practical engineering, in which two interacting oscillators (Fig.1) form two coupled nonlinear partial difference equations written as:

$$
\begin{aligned}
& m_{T} \frac{\partial^{2} y}{\partial t^{2}}+\gamma \frac{\partial y}{\partial t}-T \frac{\partial^{2} y}{\partial z^{2}}+E I \frac{\partial^{4} y}{\partial z^{4}}=\alpha_{4} \frac{\partial q}{\partial t}-\alpha_{5} \frac{\partial y}{\partial t} \\
& \frac{\partial^{2} q}{\partial t^{2}}+\left[\alpha_{1}-\alpha_{2}\left(\frac{\partial q}{\partial t}\right)^{2}\right]\left(\frac{\partial q}{\partial t}\right)+\omega_{v}^{2} q=\alpha_{3} \frac{\partial y}{\partial t}
\end{aligned}
$$

where $y(z, t)$ is cross-flow displacement of structure, $q(z, t) \quad$ is wake-oscillator describing a fluctuating variable of the near wake such as the fluctuating lift coefficient on the structure, parameters $\alpha_{1}-\alpha_{5}$ can be determined by experiments; $m_{T}=m+m_{a}$ where $m$ and $m_{a}$ are structure mass and added mass on unit length respectively; $\gamma$ is damping term of system including structure and fluid, $T$ and $E I$ are tension and bending stiffness of structure respectively;

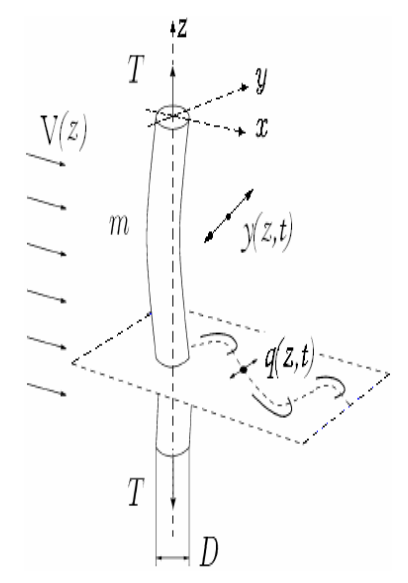

Figure 1. Wake-oscillator model 
$\omega_{v}$ is frequency of vortex shedding.

Recent researches focus on nonlinear characters of drag force and lift force $\left(\right.$ Griffin $^{[4]}$, Sarpkaya ${ }^{[5]}$ ), developing loworder approach based on consideration of interaction between structure and fluid (Facchinetti ${ }^{[6]}$ ) and synthesis model combining CFD and wake-oscillator model. In these models, added mass is an important quantity and the ideal added mass, i.e. the added mass for an accelerating body moving in an infinite, inviscid and incompressible fluid, is often adopted. For example, the ideal added mass coefficient is 0.5 for a sphere and 1.0 for a circular cylinder. However, added mass in general is variable and not equal to the ideal added mass for case of VIV.

In fact, only if the body is started from rest in a fluid otherwise at rest, the initial value of the added mass is in agreement with its ideal value because the vortices are still confined to a thin sheet on the boundary. Generally speaking, as a result of viscosity of fluid, added mass is variable in unsteady flow problems such as VIV. Sarpkaya ${ }^{[8]}$ pointed this out and did experiments to observe the fluid dynamic force (inertia component and drag component) on the body undergoing VIV. By definition, the inertia component relates to added mass in the overall lock-in range of VIV. Vikestad's experiment ${ }^{[11]}$ showed that the added mass coefficient for a circular cylinder varies from 4.5 to nearly -0.8 when the reduced velocity increases from 3.0 to 13.0 in the lock-in range of VIV. The same conclusion is also drawn based on numerical simulations (Willden and Graham $^{[12]}$ ). In this paper semi-empirical formulas for the added mass coefficient of a circular cylinder at lock-in will be given and applied to the calculation of the amplitude response of VIV with the wake-oscillator model.

Additionally, a linear relationship ${ }^{[2,3]}$ between the vibration amplitude and the reduced velocity was often utilized that can also introduce signific ant error, thus a nonlinear relationship is established in this paper. Based on these two modifications an improved wake-oscillator model for flexible riser under nonuniform current is developed. By means of empirical formula combining with iteration computation, the improved model can be conveniently programmed into computer code and has a simpler and faster computation process than CFD so as to be suitable to application of practical engineering. This model is validated by comparing it with experiment and numerical simulation. Our results show that this model can predict the response and lock-in range of VIV more accurately. At last, illustrative examples are given in which the response amplitude of flexible offshore riser experiencing lock-in vibration under action of non-uniform current is calculated and effects of riser tension and flow distribution along riser span are explored.

\section{ANALYSIS MODEL}

The variation of added mass at lock-in is investigated and estimation formula s are presented. Then a nonlinear relationship between the vibration amplitude and the reduced velocity is established. Consequently an improved analytical model of VIV is developed and its computation steps are presented.

\subsection{Variation of Added Mass at Lock-in}

For representation and simplicity a classical spring-massdamping system is taken as an example, and at lock-in the displacement and lift force are assumed to be $y(t)=y_{0} \sin \left(2 \pi f_{o} t\right) \quad$ and $\quad F_{v}(t)=F_{L} \sin \left(2 \pi f_{0} t+\phi\right)$ respectively. So the governing equation of the system subjected VIV is ${ }^{[13]}$ :

$$
\begin{gathered}
{\left[m+\frac{F_{L} \cos \phi}{y_{0}\left(2 \pi f_{0}\right)^{2}}\right] \frac{d^{2} y}{d t^{2}}+\left[2 \zeta m\left(2 \pi f_{0}\right)-\frac{F_{L} \sin \phi}{y_{0}\left(2 \pi f_{0}\right)}\right] \frac{d y}{d t}} \\
+m\left(2 \pi f_{0}\right)^{2} y=0
\end{gathered}
$$

where $f_{0}=1 / 2 \pi \sqrt{K / m}$ is the natural frequency of structure in vacuum, $K$ is stiffness of spring, $\zeta$ is damping ratio of structure. Then the added mass can be expressed as:

$$
m_{a}=F_{L} \cos \phi / y_{0}\left(2 \pi f_{0}\right)^{2}
$$

At lock-in and with low damping $(0<\zeta<<1)$, the natural frequency $f_{n}$ of system can be written as $f_{n}=\frac{f_{0}}{\sqrt{1+m_{a} / m}}$ and $m_{a}=m\left(f_{0}^{2} / f_{n}^{2}-1\right)$. So frequency ratio of fluctuation frequency $f_{\text {osc }}$ to natural frequency in static water $f_{n 0}$ can be written as:

$$
f^{*}=\frac{f_{o s c}}{f_{n 0}}=\frac{f_{n}}{f_{n 0}}=\sqrt{\frac{m+m_{D}}{m+m_{a}}}
$$

Where $m_{D}$ is the displaced mass. By further consideration of the function relationship describing $f^{*}$ varying with reduced velocity $V_{r}=V / f_{n 0} D$ ( $V$ is flow velocity ) given by Williamson $^{[14]}$, the calculation formulas of added mass coefficient can be given for two cases, mass ratio $m^{*}<10.0$ and $m^{*} \geq 10^{[13]}\left(m^{*}=m / m_{D}\right)$.

1) Mass ratio $m^{*}<10$

The added mass coefficient can be written as :

$$
\begin{aligned}
& C_{a}=\frac{m^{*}+1}{G^{2}}-m^{*}, \text { when } 5.0 \leq V_{r}<5.75 \bar{m} \\
& C_{a}=-0.54, \quad \text { when } 9.25 \bar{m}>V_{r}>5.75 \bar{m}
\end{aligned}
$$

$$
\begin{aligned}
& \text { where } \quad G=\frac{\bar{m}-1.0}{5.75 \bar{m}-5.0}\left(\frac{V}{f_{0} D} \sqrt{1+\frac{1}{m^{*}}}-5.0\right)+1.0 \\
& \bar{m}=\sqrt{\frac{m^{*}+1.0}{m^{*}-0.54}} ;
\end{aligned}
$$


2) Mass ratio $m^{*} \geq 10$

Since $\sqrt{\left(m+m_{D}\right) /\left(m+m_{a}\right)}=\bar{m}$, we have:

$$
C_{a}=-0.54
$$

It should be pointed out that Eqs. (6) and (7) should be used under the following conditions: $m^{*}>-0.54$ 、 $10^{5}>\operatorname{Re}>300$ and $\left(m^{*} \zeta\right) \leq 0.02$. For the verification of estimation formulas, Eqs. (6) and (7) are compared with experiments and numerical simulations respectively shown in Fig.2 and Fig.3. It can be seen that Eqs. (6) and (7) agree with the experiments results and numerical simulations well, especially at lower reduced velocity.

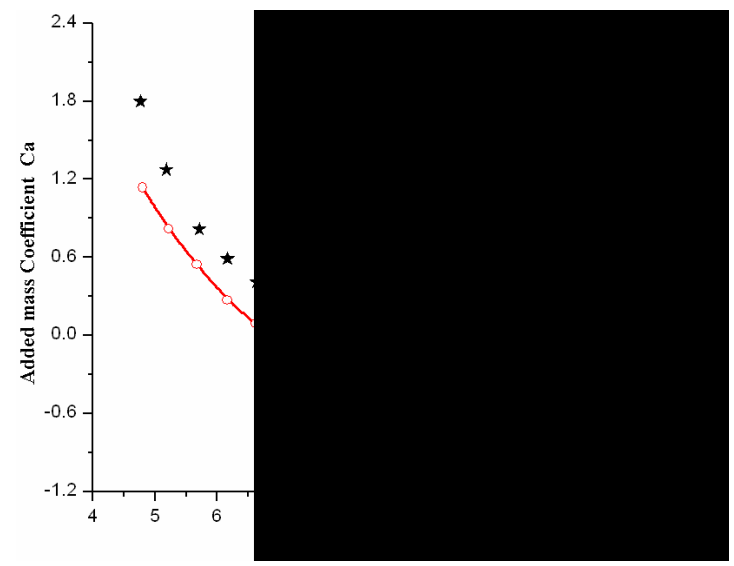

Figure 2. Comparison between calculation result and experimental result ( $m^{*}=0.6366$ ).

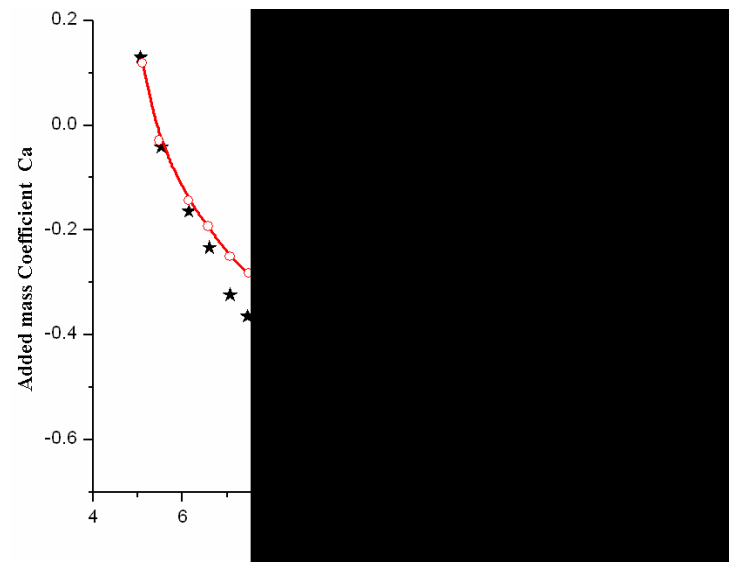

Figure 3. Comparison between calculation result and numerical simulations $\left(m^{*}=1.6552\right)$.

\subsection{Improved Wake-oscillator Model}

A linear relationship between vibration amplitude and reduced velocity was utilized in old model ${ }^{[3]}$ that can introduce a significant difference form reality of VIV and its errors defined as the relative errors of calculation values to experiment values (or numerical simulation values) are shown in Tab.1. Observing experimental results $\left(\right.$ Feng $^{[15]}$ and Brika ${ }^{[16]}$ ) and numerical simulation (Foulhoux ${ }^{[17]}$ ) we can see that it is more reasonable considering a nonlinear relationship (shown in Fig.4). Thus a nonlinear relationship is established. And the relationship function is described as follow:

$$
\frac{y}{D}=\bar{y}_{0}+\frac{Y}{w \sqrt{\pi / 2}} e^{-2\left(V_{r}-V_{r}^{c}\right)^{2} / w^{2}}
$$

The regression parameters based on experiments and numerical simulations are $\bar{y}_{0}=0.014, Y=2.040, V_{r}^{c}=6.172$ and $w=1.723$. The errors introduced by the nonlinear relationship compared with experimental results and numerical simulation are also listed in Tab. 1. It can be seen that errors of nonlinear relation are significantly smaller than errors of linear relation.

Table 1. Errors of two relationships compared with experiments and numerical simulations

\begin{tabular}{ccccc}
\hline Error & Griff & Feng & Brika & Foulhoux \\
\hline Nonlinear & .1125 & .1029 & .1001 & .0898 \\
Linear & .1539 & .2396 & .2183 & .1977 \\
\hline
\end{tabular}

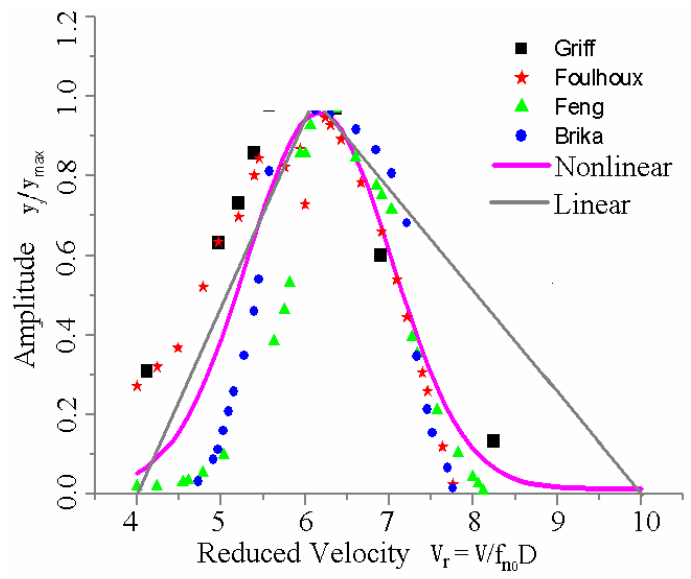

Figure 4. Relationship between amplitude and reduced

$$
\text { velocity }
$$

By now the algorithm of the improved wake-oscillator model is summarized as follows:

1) Carry out the added mass under given flow velocity according to formula 6) (or (7)) and consequently natural frequencies and mode shape. Then obtain lock-in region distributions along the riser span of the lock-in modes.

2) Carry out mode effect mass $m_{n}$ and shape coefficient $I_{n}$ of the $n$th lock-in mode. Here the mode effect mass is

$$
m_{n}=\int_{0}^{l} m(x) \xi_{n}^{2}(x) d x / \int_{0}^{l} s(x) \xi_{n}^{2}(x) d x
$$


and

mode

shape

coefficient

$I_{n}=\int_{0}^{l} m(x) \xi_{n}^{4}(x) d x / \int_{0}^{l} m(x) \xi_{n}^{2}(x) d x$, where $\xi_{n}$ is the

$n$th mode shape, $s(x)$ is a lock-in parameter which has value of 1.0 in lock-in region and 0.0 in non-lock-in region along the riser span.

3) Carry out system damping ratio $\zeta_{n}^{S}$ and amplification factor $F_{n}$ by iteration as follow:

a) Assume the initial value of structure damping $\zeta_{n} I$.

b) Calculate the amplification factor $F_{n}=1 /\left[1+9.6\left(\mu_{r}^{n} \zeta_{n}^{s}\right)^{1.8}\right]$, where $\mu_{r}^{n}=m_{n} / m_{D}$ is the mass ratio of the $n$th mode.

c) Calculate the system damping ratio $\zeta_{n}^{S}=\zeta_{n}^{I}+F_{n} \phi_{n}$, where the effective damping

$$
\phi_{n}=\frac{2 D \int_{0}^{l} C_{D}(x) \rho D(x)[1-s(x)]\left|\xi_{n}(x)\right|^{3} d x}{3 \pi\left[\int_{0}^{l} m(x) \xi_{n}^{4}(x) d x\right]^{1 / 2}\left[\int_{0}^{l} m(x) \xi_{n}^{2}(x) d x\right]^{1 / 2}}
$$

where $C_{D}$ is drag coefficient and generally has a constant value of 1.2

d) Check if the system damping ratio $\zeta_{n}^{S}$ and amplification factor $F_{n}$ satisfy the demand of convergence. If no, set $\zeta_{n}^{I}=\zeta_{n}^{S}$ and go back to step b); if yes, stop iteration.

4) Calculate the amplitude of response using following formula:

$$
Y_{n}(x)=D F_{n} I_{n}^{-1 / 2} \xi_{n}(x)
$$

5) Modify the amplitude according to the reduced velocity employing the non-linear relationship given by Eq.(8), and carry out mode stress.

Repeat calculation from steps 1) to 5) for every lock-in mode.

Amplitudes of VIV response of a cylinder ${ }^{[18]}$ are predicted using the presented model and is plotted against flow velocity (shown in Fig. 5), and they are compared with the corresponding experimental results (Khalak ${ }^{[18]}$ ) so as to verify the presented model. Figure 5 shows that the predicted responses agree with the experimental results well. As a comparison, the responses calculated using the original model (without improvements presented in this paper) are also plotted in Fig. 5. It shows that the improved model presented in this paper is more accurate than the original model.

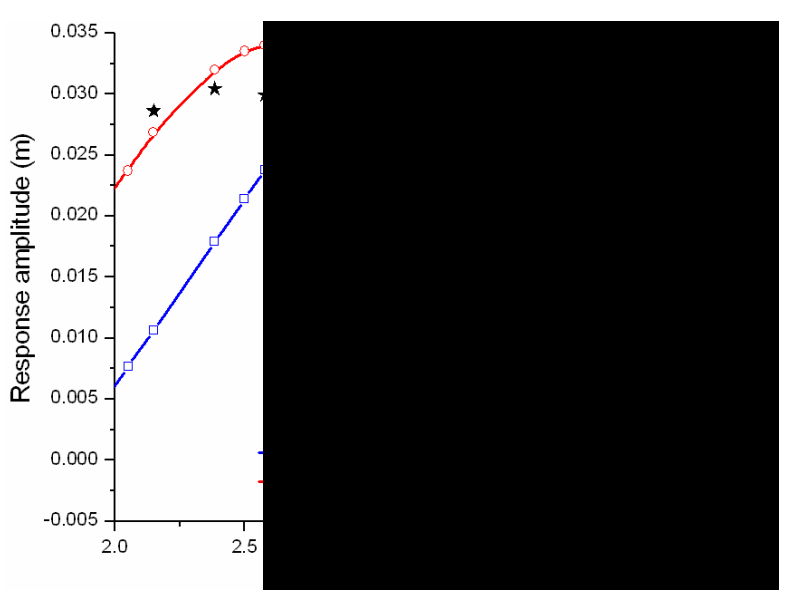

(a) $m^{*}=2.4, \zeta=0.0045$

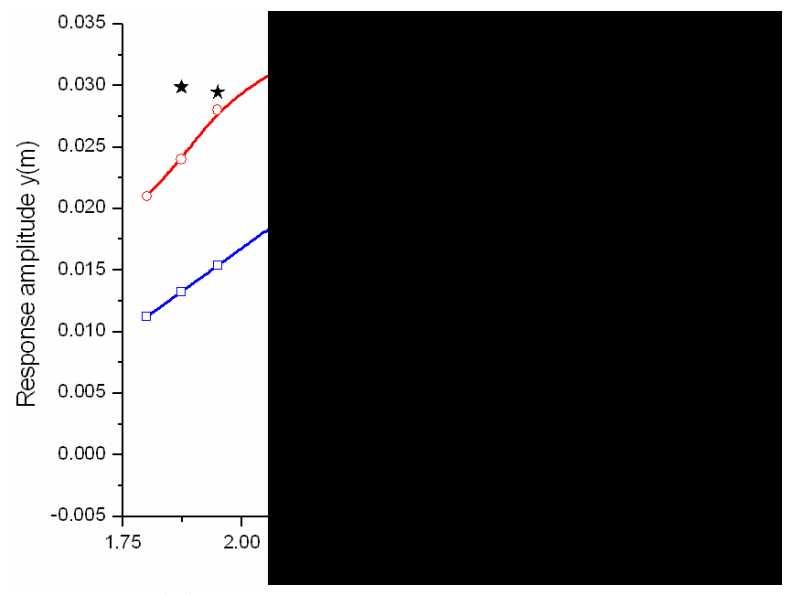

(b) $m^{*}=10.1, \zeta=0.00134$

Figure 5. Comparison of amplitude response between calculation and experiment.

\section{APPLICATIONS AND DISCUSSIONS}

\subsection{Effect of Tension on Response of VIV}

The presented model is applied to predict VIV response of flexible tension cable of Conoco's Huttonn TLP (Tension Leg Platform) with structure property characters as : $300 \mathrm{~m}$ length, $1.1176 \mathrm{~m}$ diameter, $0.038 \mathrm{~m}$ wall thickness; $1000 \mathrm{Kg} / \mathrm{m}$ mass per unit length, $3.854 \times 10^{6} \mathrm{KN} \mathrm{m}^{2}$ bending stiffness; and a shear current is assumed as a function of $z$ having form of $V(z)=0.4+0.008 z \quad(\mathrm{~m} / \mathrm{s})$, positive direction of coordinate $z$ axis vertically directs from sea bed to sea surface and at sea bed $z=0 \mathrm{~m}$. Effect of cable tension on the response of VIV is explored. Here the maximum tension $T_{\max }=2.1 \times 10^{7} \mathrm{~N}$ and tension force along cable is assumed to be constant.

Figure 6 presents the amplitude of response with tension ratio $T / T_{\max }$ ranging from 0.1 to 1.0 . It is interesting that the total response firstly decreases and approaches to a minimum value $y / D=1.06$ at $T / T_{\max }=0.55$ and then the total 
response increases as tension ratio increases, though the stiffness increases monotonically with increase of tension ratio. Mode responses of four lock-in modes are also plotted in Fig.6. It shows that mode response behaves in different way, some increase monotonically (e.g. the 1st mode), some decrease monotonically (e.g. the 4th mode) and some vary not monotonically (e.g. the 2nd mode) as tension ratio increases.

Figure 7 shows the variation of lock-in region distributions, as form of region width (lock-in region is determined according to the reduced velocity ranging from 4 to 10) and location along cable span, of lock-in modes versus cable tension force. In fact it virtually influences mode response. Generally speaking, when lock-in region is wider and nearer to the anti-node of mode shape, displacement of response is larger, because the effective mass (Eq.s (9)) and effective damping (Eq. (10)) of lock-in mode become smaller, and vice versa. Another thing should be noted is that there exists a minimum value of response that is favorable for structure safety and fatigue life, or it is a desired status to platform designer

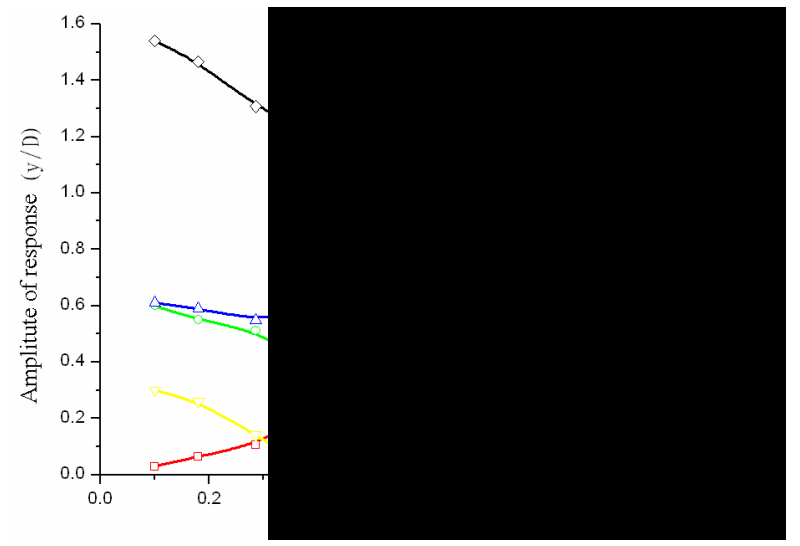

Figure 6. Effect of tension force on amplitude of response

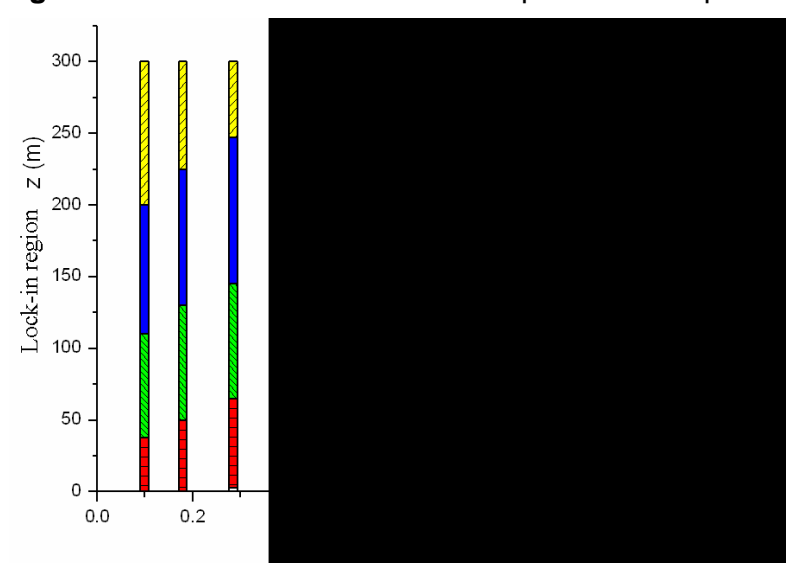

Figure 7. Effect of tension force on lock-in region distribution

\subsection{Effect of Flow Distribution on Response of VIV}

A segmented and varying along span of riser flow distribution shown in Fig. 8 is considered to examine effect of flow distribution on response of VIV. The ratio of the maximum amplitude of response for segmented flow to the amplitude of response for uniform flow over the entire riser is given in Fig.9 for the lowest three modes as a function of the extent of the flow and internal structure damping (0.008、0.02、0.10). And also the mode stress results are shown in Fig.10. The riser property parameters are the same as in section 3.1.

Figure 9 shows that amplitude of response of three

Figure 8. Scheme of flow distribution modes increases monotonically as flow distribution becomes wider, though slope of curve behaves differently. Since the effective mass and damping of system become smaller as flow segment becomes wider and consequently lock-in region becomes wider, amplitude of response becomes larger. And similarly, for case of lock-in region is nearer to the anti-node of mode shape the slope of curve is larger because of the decrease of effective mass and damping and the consequent lower efficiency of energy absorbing, and vice versa.

Figure 9 also shows that curve with smaller damping is smoother, or variation of curve slope is smaller, than that with larger damping. It means that the sensitivity of VIV response to the location of lock-in region is reduced with increase of damping because the effective damping becomes larger.

Mode stress of response has similar behavior with amplitude of response except one point that for most cases in this example stress of higher mode has a larger value than lower mode and should be paid attention for analysis of structure fatigue life.

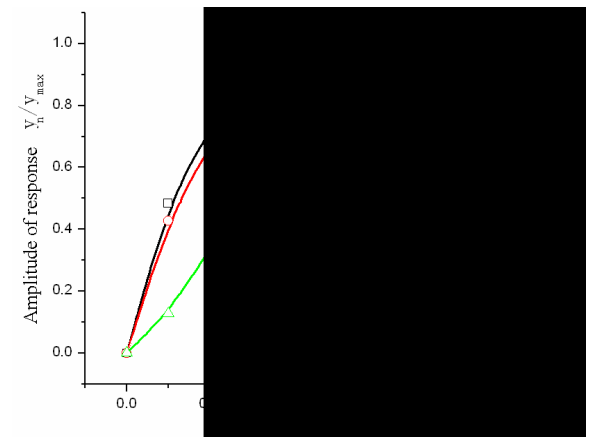

(a) Response of 1st mode 


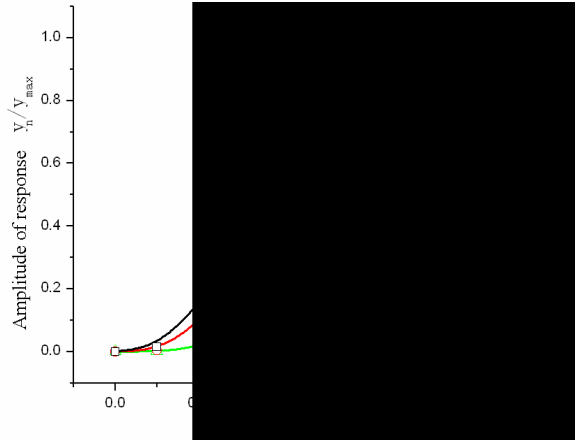

(b) Response of 2nd mode



(c) Response of 3rd mode

Figure 9. Amplitude ratio of response

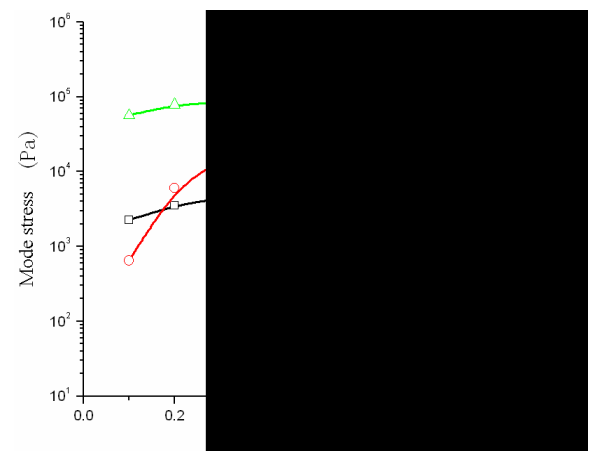

(a) Damping ratio is 0.008

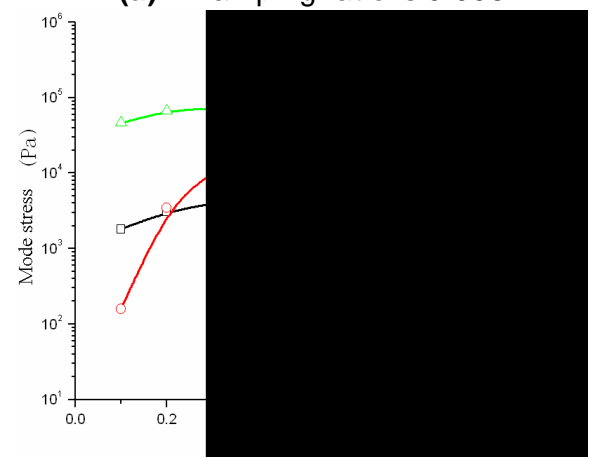

(b) Damping ratio is 0.02

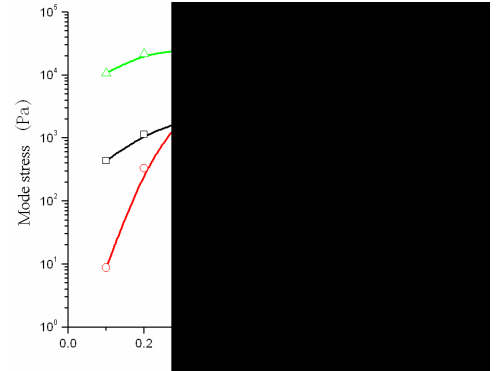

(c) Damping ratio is 0.10

Figure 10. Mode stress of response

\section{CONCLUSIONS}

Based on improving the wake oscillator model, an analytical model for response of VIV of flexible riser under nonuniform current is presented, in which the variation of added mass and the nonlinear relationship between amplitude of response and reduced velocity are considered. And the improved analytical model is validated by comparing it with experiments and numerical simulations. Our results show that the improved model can predict response of VIV and lock-in region more accurately. Illustrative examples in which response of flexible riser experiencing lock-in vibration under action of non-uniform current is calculated and we draw following conclusions:

1) Riser tension influences he total amplitude of VIV response by variation of lock-in region distribution. When lockin region is wider and nearer the anti-node mode shape, displacement response is larger because of decrease of the effective mass and damping of system, and vice versa. It should be noted that there exists a minimum value of response that is favorable for structure safety and fatigue life.

2 ) Amplitude of response of lock-in mode increases monotonically as flow distribution becomes wider. But slope of curve behaves differently. Since the effective mass and damping of system become smaller as flow segment becomes wider and consequently lock-in region becomes wider. When lock-in region is near anti-node of mode shape, the slope of curve becomes larger because of decrease of effective mass and damping, and vice versa.

3) The sensitivity of VIV response to the location of lockin region is reduced with increase of damping because the effective damping becomes larger. For most cases in the example stress of higher mode has a larger value than lower mode and should be paid attention for analysis of fatigue life.

\section{ACKNOWLEDGMENTS}

The authors would like to acknowledgement supports from the National Natural Science Foundation of China (10772183, 10532070) and 863 Program (2006AA09A103-4, 2006AA09Z301).

\section{REFERENCES}

[1] Hartlen R T, Currie I G, 1970, "Lift-Oscillator Model of VotexInduced Vibration", Journal of the Engineering 
Mechanics, Vol.96(5), pp. 577-591.

[2] Iwan W D, 1981, "The Vortexinduced Oscillation of Nonuniform Structure Analysis", Journal of Sound and Vibration, Vol. 79(2), pp. 291-301.

[3] Lyong G J, Patel M H, 1986, “A Prediction Technique for Vortex Induced Transverse Response of Marine Risers and Tethers", Journal of Sound and Vibration, Vol. 111(3), pp. 467-487.

[4] Griffin O M, Skop R A, Ramberg S E, 1975, "The Resonant Vortex Excited Vibrations of Structures and Cable Systems", Proceedings of Offshore Technology Conference, Houston, Texas, OTC2319.

[5] Sarpkaya T, 1977, "In-Line and Transverse Forces on Cylinders in Oscillatory Flow at High Reynolds Numbers", Journal of Ship Research, Vol. 21, pp. 200216.

[6] Facchinetti M L, Langre E, 2004, "Coupling of structure and wave oscillators in VIV", Journal of Fluids and structures, Vol. 19, pp. 123-140.

[7] Pan Z Y, Cui W C, Liao Q M, 2006, “A prediction model for VIV of a slender marine riser", Journal of Ship Mechanics, Vol.10(3), pp. 115-12. (in Chinese)

[8] Sarpkaya T, 2004, "A Critical review of the intrinsic nature of vortex-induced vibration". Journal of Fluids and Structures Mechanics, Vol. 46, pp. 389-447.

[9] Larsen C M, Halse K H, 1997, "Comparison of models for vortex induced vibrations of slender marine structures", Marine Structures, Vol. 10, pp. 413-441.

[10] Cunff C, Biolley F, 2002, "Vortex-Induced Vibrations of Risers: Theoretical, Numerical and Experimental Investigation", Oil \& Gas Science and Technology - Rev. IFP, Vol. 57(1), pp. 59-69

[11] Vikestad K, Vandiver J K, Larsen C M, 2000, “Added mass and oscillation frequency for a circular cylinder subjected to vortex-induced vibrations and external disturbance", Journal of Fluids and Structures, Vol.14, pp. 1071-1088.

[12] Willden R H J and Graham J M R, 2001, "Numerical prediction of VIV on long flexible circular cylinders", Journal of Fluids and Structures, Vbl. 15, pp. 659 669.

[13] Wang Y, Chen W M, Lin M. 2007, "Study on the Variation of Added Mass and Its Application to the Calculation of Amplitude Response for a Circular Cylinder at Lock-in". China Ocean Engineering, Vol. 21(3), pp. 429-37.

[14] Williamson C, Govardhan R. 2004, "Vortex-induced vibration", Annual Review of Fluid Mechanics, Vol. 36, pp. 413-455.

[15] Feng CC, 1968, "The measurement of vortex induced effects in flow past stationary and oscillating circular and d-section cylinders", Master's Thesis, Department of Mechanical Engineering, The University of British Columbia, Canada

[16] Brika D, Laneville A, 2002, "Vrtex-induced Vibrations of a long flexible circular cylinder", Journal of Fluid Mechanics, Vol. 250, pp. 481-508.

[17] Foulhoux L, Saubestre V, 1994, “An Engineering Approach to Characterize the Look-in Phenomenon Generated by a current on a Flexible Column", International Journal of Offshore and Polar Engineering, Vol. 4(3), pp. 231-233.

[18] Khalak, A, Williamson C, 1997, "Fluid forces and dynamics of a hydroelastic structure with very low mass and damping", Journal of Fluids and Structure, Vol. 11, pp. 973 982.

[19] de Oliveria J, Fjield S, 1988, "Concrete hulls for tension leg platforms", Proceedings of $20^{\text {th }}$ Offshore technology Conference, OTC5636, Houston.

[20] Wang DY, Ling G C, 1998, "Vortex-induced nonlinear vibrations of TLP tethers under circumstances of platform oscillation", ACTA Oceanologica SINCA, Vol. 20(5), pp. 119-128. (in Chinese) 